\title{
ENSAYO DE COMPACTACIÓN GIRATORIA EN SUELOS COMO ALTERNATIVA AL ENSAYO DE COMPACTACIÓN PROCTOR
}

\section{GYRATORY COMPACTION TEST IN SOILS AS ALTERNATIVE TO THE PROCTOR COMPACTION TEST}

\author{
Javier Fernando, Camacho Tauta \\ Ing. Civil MSc, Profesor Asistente, Facultad de Ingeniería, Investigador Grupo de Geotecnia, jcamacho@umng.edu.co \\ Oscar Javier, Reyes Ortiz \\ Ing. Civil MSc, Profesor Asociado, Facultad de Ingeniería, Investigador Grupo de Geotecnia, oreyes@umng.edu.co \\ Dolly Fernanda, Méndez González \\ Ing. Civil, Asistente de Investigación, Facultad de Ingeniería, Grupo de Geotecnia, dolly.mendez@umng.edu.co \\ Fecha de recepción: Septiembre 12 de 2007 \\ Fecha de aprobación: Noviembre 13 de 2007
}

\section{RESUMEN}

El propósito de este trabajo es analizar experimentalmente una metodología alternativa para el proceso de compactación de suelos en laboratorio. Tradicionalmente se ha utilizado el método conocido como "compactación proctor", aunque siempre se ha reconocido que no representa fielmente las condiciones en que se produce la compactación en el campo. En el ensayo de compactación giratoria, el proceso se produce por amasado, lo cual asemeja mejor el efecto de los equipos de vibrocompactación en obra.

Se realizaron diferentes ensayos con presiones verticales entre 100 y $400 \mathrm{kPa}$, se mantuvo constante el ángulo de giro $\left(1.25^{\circ}\right)$ y el material se ensayó con diferentes contenidos de humedad. Con los resultados de los ensayos se determinó que para obtener una curva de compactación similar a la conseguida en el ensayo de proctor modificado se pueden utilizar dos opciones, la primera es trabajar con una presión vertical de $400 \mathrm{kPa}, 100$ giros y ángulo de $1.25^{\circ}$ y la segunda opción es aplicar una presión $350 \mathrm{kPa}, 300$ giros y un ángulo de $1.25^{\circ}$.

PALABRAS CLAVE: Compactador giratorio, ensayo de compactación proctor, peso específico seco máximo, humedad óptima. 


\section{ABSTRACT}

The purpose of this work is to analyze an alternative method for soil compaction in laboratory. The standard laboratory test is known as "Proctor Test", although research works have recognized that this method does not represent faithfully the real field conditions. In the gyratory compaction test, the process is produced by kneaded which resembles better the effect of the vibratory construction machines.

Different tests were performed with vertical pressures between $100 \mathrm{kPa}$ and $400 \mathrm{kPa}$, maintaining constant the twist angle $\left(1.25^{\circ}\right)$. The material has been tested in different moisture conditions. The results have shown that there are two options to obtain a curve similar to the proctor test. The first way is to work with $400 \mathrm{kPa}$ vertical pressure, 100 turns and $1.25^{\circ}$ twist angle. The second way is to apply a $350 \mathrm{kPa}$ vertical pressure, 300 turns and $1.25^{\circ}$ twist angle.

KEY WORDS: Gyratory testing machine, Proctor compaction test, maximum dry unit weight, optimum moisture.

\section{INTRODUCCIÓN}

Para reducir el porcentaje de vacíos de un suelo y mejorar sus propiedades mecánicas como la resistencia al corte, la compresibilidad y el potencial de expansión, se realizan diferentes procedimientos o ensayos de compactación de suelos, logrando conseguir una estructura de máxima densidad que presenta deformaciones mínimas cuando se somete a diferentes cargas.

En materiales granulares, el estado de compactación del suelo se determina por medio del parámetro densidad relativa, la cual está en función del peso unitario seco máximo, peso unitario seco mínimo y el peso unitario seco in- situ [1], de acuerdo con la Ecuación 1.

$$
\operatorname{Dr}=\frac{\gamma_{d}-\gamma_{d \min }}{\gamma_{d \max }-\gamma_{d \min }} * \frac{\gamma_{d \max }}{\gamma_{d}}
$$

El peso unitario seco que puede alcanzar el material está relacionado con la cantidad de energía aplicada, el método de aplicación, el volumen de material compactado, la densidad de las partículas y el contenido de humedad del suelo. Los ensayos de compactación en laboratorio buscan establecer la relación entre el peso unitario seco y el contenido de humedad para un determinado tipo de suelo (i.e. densidad de partículas) y un proceso de compactación determinado (i.e. volumen de suelo y cantidad y forma de aplicación de la energía de compactación). Está relación permite determinar el contenido de humedad óptimo con el que el suelo debe ser compactado en obra, para que se pueda logar una densidad o peso unitario máximo.

Se utilizan varios métodos de compactación en campo como por ejemplo la compactación vibratoria, la compactación por amasado y la compactación estática. Para evaluar el por- 
centaje de compactación obtenido en campo, es necesario compararlo con los resultados obtenidos en laboratorio. Los procedimientos de laboratorio más conocidos son los ensayos de proctor estándar y proctor modificado (Normas INV E- 141 e INV E- 142, respectivamente [8]). Estos procedimientos, sin embargo no son rigurosamente comparables con los procedimientos equivalentes aplicados en campo, principalmente porque la forma de compactación varía considerablemente. Mientras que en los ensayos de compactación proctor se aplica una forma de energía por impacto, en el campo el amasado es la forma de transferencia de energía aplicada al suelo. Las energías de compactación aplicadas en obra tienden a ser mayores que las que se aplican en los ensayos de proctor en el laboratorio [6]. Es por esto que se han llevado a cabo diferentes estudios sobre el tema de compactación de suelos en la búsqueda de diferentes alternativas de compactación en el laboratorio. Un ejemplo de esto, es el trabajo realizado por el Departamento de Transporte de Florida en el año 2003, en el cual se evaluaron las ventajas de la compactación del suelo en un equipo de compactación giratoria [12]

El uso del compactador giratorio se inició en 1930, mientras que los trabajos de investigación desarrollados durante las siguientes décadas fueron enfocados a los diseños de cementos y mezclas asfálticas. En el año de 1987 surgió el programa SHRP (Strategic Highway Research Program), que en la actualidad se conoce como la metodología Superpave (Superior Performing Asphalt Pavement), sistema de diseño que se utiliza para establecer el desempeño de las mezclas asfálticas.

Para la década de 1990, el Departamento de Transporte del estado de Texas inició trabajos de investigación en suelos con el equipo de compactación giratoria. En este trabajo se encontró que los ensayos Proctor, que comúnmente se realizan en el laboratorio, presentan resultados lejanos a los obtenidos en la compactación en campo, caso contrario ocurrió con los ensayos de compactación de suelos en el compactador giratorio.

Este artículo presenta los resultados obtenidos en los ensayos de compactación giratoria de un suelo granular normalmente utilizado como subbase granular en las vías de la ciudad de Bogotá y una comparación con las curvas de los ensayos de proctor modificado y proctor estándar. Esta comparación permitirá evaluar que parámetros del ensayo de compactación giratoria deben establecerse para obtener la densidad seca máxima del material.

\section{MARCO CONCEPTUAL}

\subsection{COMPACTACIÓN DEL SUELO}

La aplicación de energía a un espécimen de suelo, con el fin de incrementar su densidad o peso seco unitario y reducir el volumen de vacíos, es lo que se conoce como compactación del suelo. La compactación del suelo aporta a este las siguientes ventajas [7]:

- Aumenta la resistencia al corte y así mismo mejora la capacidad de soporte de vías y subestructuras. 
- $\quad$ Reduce o previene la compresibilidad y los asentamientos.

- Reduce el volumen de vacíos

- Permite reducir y controlar los potenciales de expansión, contracción y expansión por congelamiento

Para conocer el porcentaje de compactación del suelo en campo es necesario que previamente se haya determinado la densidad seca máxima de éste, por medio de la ejecución de un ensayo o prueba de laboratorio en el que se mantenga una energía constante. Estos ensayos permiten obtener una relación entre el peso seco unitario y el contenido de humedad (ver figura 1), además conduce a establecer el contenido de humedad con el que se debe compactar el suelo para que se logre alcanzar el peso unitario seco máximo [6].

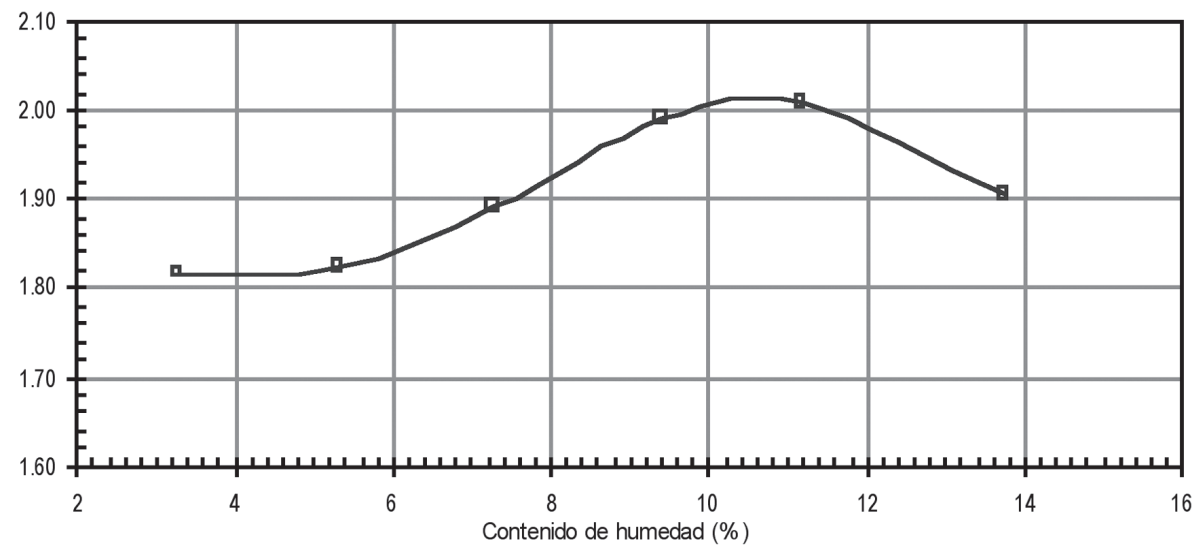

Figura 1. Peso unitario seco en función del contenido de humedad. Resultados típicos obtenidos en un ensayo de compactación proctor.

La relación entre el contenido de humedad del suelo $(\omega)$, el peso unitario seco $\left(\gamma_{d}\right)$ y el peso unitario húmedo $\left(\gamma_{h}\right)$, se presenta en la Ecuación 2, la cual es requerida para los cálculos de cualquier ensayo de compactación.

$$
\gamma_{\mathrm{d}}=\frac{\gamma_{\mathrm{h}}}{1+\omega}
$$

\subsection{MÉTODOS UTILIZADOS EN CAMPO}

Compactación estática: en este tipo de compactación se utilizan equipos conocidos como rodillos lisos, neumáticos y de tractor; el tambor de estos equipos algunas veces se puede llenar con agua y arena, lo que incrementa el peso del equipo y por ende también incrementa la presión ejercida sobre el suelo, las presiones ejercidas por el equipo pueden alcanzar $400 \mathrm{kN} / \mathrm{cm}^{2}$. Este método se utiliza en suelos bien gradados que tengan aproximadamente el $20 \%$ de finos [5]. 
Compactación por amasado: para este caso se utiliza el rodillo pata de cabra (Figura 2), las presiones de compactación que se obtienen por este método están entre $1500 \mathrm{kN} / \mathrm{cm}^{2}$ y $7500 \mathrm{kN} / \mathrm{cm}^{2}$. Este método es ideal para la compactación de suelos arcillosos porque produce mayores esfuerzos de cizallamiento en toda la masa de la capa de suelos por compactar, concentrando grandes presiones en ciertos puntos; gracias a la presencia de los vástagos que concentran gran energía en áreas pequeñas y se penetran en el interior de la capa de suelo [10].

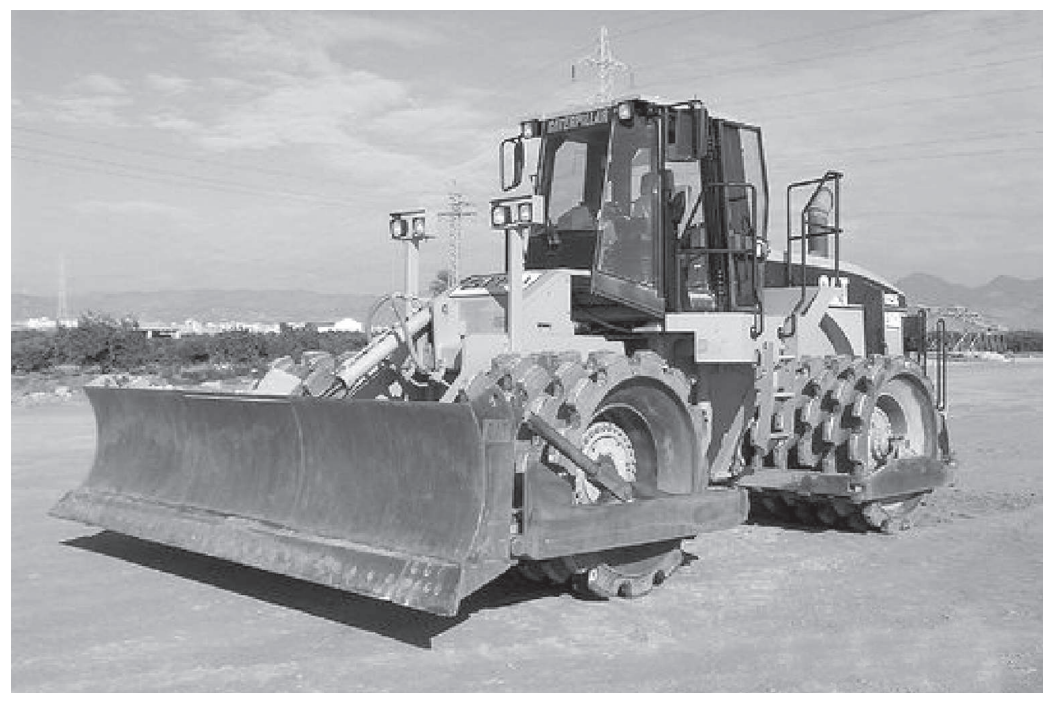

Figura 2. Compactador pata de cabra.

Compactación por vibración: en este método se pueden utilizar equipos como los descritos en los métodos anteriores, solo que para este caso, el equipo cuenta con un efecto especial de vibración. Dentro de los equipos utilizados en este caso se encuentra los rodillos tipos tramper, vibrocompactadores, canguros y pisones. Es muy común que los canguros y pisones sean utilizados en áreas pequeñas [5].

\subsection{MÉTODOS UTILIZADOS EN LABORATORIO}

Compactación por impacto: también conocido como método dinámico, consiste en colocar una cantidad de material con cierto contenido de humedad en un molde cilíndrico y compactarlo con un determinado número de golpes por capa. La cantidad de energía aplicada a cada ensayo se determinada por medio de la ecuación 3.

$$
E=\frac{n \cdot N \cdot W \cdot h}{v}
$$


Siendo:

$N=$ número de capas, $n=$ número de golpe, $W=$ Peso del martillo, $h=$ altura de caída del martillo y $v=$ Volumen del molde.

Los ensayos más utilizados son el proctor estándar y el proctor modificado, que se rigen por normas estadounidenses, sin embargo, también existen ensayos fundamentados en normas británicas cuyas especificaciones se muestran en la Tabla 1 [2].

Tabla 1. Especificaciones de los ensayos de compactación en laboratorio.

\begin{tabular}{|c|c|c|c|c|c|c|}
\hline \multirow[b]{2}{*}{ Ensayo } & \multirow{2}{*}{$\begin{array}{l}\text { Referencia } \\
\text { Estándar }\end{array}$} & \multicolumn{2}{|c|}{ Martillo } & \multirow{2}{*}{$\begin{array}{c}\text { Volumen } \\
\text { del } \\
\text { molde }\end{array}$} & \multirow[b]{2}{*}{ Capas } & \multirow[b]{2}{*}{$\begin{array}{c}\text { Golpes } \\
\text { por capa }\end{array}$} \\
\hline & & Masa & $\begin{array}{l}\text { Altura de } \\
\text { caída }\end{array}$ & & & \\
\hline $\begin{array}{l}\text { Proctor } \\
\text { estándar } \\
\text { AASHTO } \\
\text { estándar }\end{array}$ & $\begin{array}{l}\text { ASTM D - } 698 \\
-78 \text { ASSHTO } \\
\text { T -99 }\end{array}$ & $\begin{array}{l}2.49 \mathrm{Kg} . \\
(5.5 \mathrm{lb} .)\end{array}$ & $\begin{array}{l}305 \mathrm{~mm} \\
\text { (12 pul) }\end{array}$ & $944 \mathrm{~cm}^{3}$ & 3 & 25 \\
\hline $\begin{array}{l}\text { Proctor } \\
\text { estándar } \\
\text { AASHTO } \\
\text { modificado }\end{array}$ & $\begin{array}{l}\text { ASTM D } \\
-1557-78 \\
\text { ASSHTO T } \\
-180\end{array}$ & $\begin{array}{c}4.54 \mathrm{Kg} . \\
(10 \mathrm{lb} .)\end{array}$ & $\begin{array}{l}457 \mathrm{~mm} \\
\text { (18 pul) }\end{array}$ & $944 \mathrm{~cm}^{3}$ & 5 & 25 \\
\hline \multirow{2}{*}{$\begin{array}{c}\text { Proctor } \\
\text { estándar } \\
\text { Normas } \\
\text { INVIAS } \\
\text { MÉTODO A * }\end{array}$} & $\begin{array}{l}\text { INV E-141 } \\
\text { Método A y } \\
\text { Método C }\end{array}$ & $\begin{array}{l}2.5 \mathrm{Kg} . \\
(5.5 \mathrm{lb} .)\end{array}$ & $\begin{array}{l}305 \mathrm{~mm} \\
\text { (12 pul) }\end{array}$ & $817 \mathrm{~cm}^{3}$ & 3 & 25 \\
\hline & $\begin{array}{l}\text { INV E - } 141 \\
\text { Método B y } \\
\text { Método D }\end{array}$ & $\begin{array}{l}2.5 \mathrm{Kg} . \\
(5.5 \mathrm{lb} .)\end{array}$ & $\begin{array}{l}305 \mathrm{~mm} \\
\text { (12 pul) }\end{array}$ & $1815 \mathrm{~cm}^{3}$ & 3 & 56 \\
\hline $\begin{array}{c}\text { Proctor } \\
\text { Modificado } \\
\text { Normas } \\
\text { INVIAS } \\
\text { MÉTODO A * }\end{array}$ & $\begin{array}{l}\text { INV E - } 142 \\
\text { Método A y } \\
\text { Método C }\end{array}$ & $\begin{array}{c}4.54 \mathrm{Kg} . \\
(10 \mathrm{lb} .)\end{array}$ & $\begin{array}{l}457 \mathrm{~mm} \\
\text { (18 pul) }\end{array}$ & $817 \mathrm{~cm}^{3}$ & 5 & 25 \\
\hline $\begin{array}{l}\text { Proctor } \\
\text { estándar } \\
\text { Normas } \\
\text { INVIAS } \\
\text { MÉTODO B }\end{array}$ & $\begin{array}{l}\text { INV E - } 142 \\
\text { Método B y } \\
\text { Método D }\end{array}$ & $\begin{array}{c}4.54 \mathrm{Kg} . \\
(10 \mathrm{lb} .)\end{array}$ & $\begin{array}{l}457 \text { mm } \\
\text { (18 pul) }\end{array}$ & $1815 \mathrm{~cm}^{3}$ & 5 & 25 \\
\hline $\begin{array}{c}\text { MARTILLO } \\
\text { VIBRATORIO }\end{array}$ & $\begin{array}{l}\text { BS } 1377: \\
1975 \text { Ensayo } \\
14\end{array}$ & \multicolumn{5}{|c|}{$\begin{array}{l}\text { Se utiliza un molde CBR para compactar una muestra } \\
\text { aproximadamente } 2360 \mathrm{~cm}^{3} \text { de volumen mediante el uso de } \\
\text { un martillo vibratorio; el suelo se compacta en tres capas } \\
\text { iguales mediante vibración. }\end{array}$} \\
\hline * Nota: & sc & $\begin{array}{l}\text { norma } \\
\text { amañc }\end{array}$ & $\begin{array}{l}-141 y \\
\text { o del m }\end{array}$ & $-142, e$ & todo & ende \\
\hline
\end{tabular}


Otro de los ensayos que se encuentran en la Tabla 1, es el del método del martillo vibratorio, que consiste la aplicación de una presión estática y vibración al suelo durante un tiempo de 60 segundos, a tres capas iguales de suelo.

Harvard Miniatura: este método de laboratorio es usado en materiales finos, plásticos y con partículas menores a $2 \mathrm{~mm}$, fue desarrollado por el Profesor S.D Wilson, en la Universidad de Harvard. Consiste en la compactación del suelo por medio de presión, para lo cual se requiere una cámara cilíndrica metálica de $3.3 \mathrm{~cm}$ de diámetro y $7.2 \mathrm{~cm}$ de altura, el molde está provisto de una extensión removible de $3.5 \mathrm{~cm}$ de altura y además de una barra metálica de $1.3 \mathrm{~cm}$ de diámetro, que actúa como un émbolo y aplica presión [9].

Compactación giratoria: este método consiste en la aplicación de acciones simultáneas como la presión vertical y la acción giratoria, con un ángulo de inclinación con respecto al eje vertical, ejercidas por el equipo, asemejando los procesos constructivos en obra.

Las primeras compactaciones con este equipo se desarrollaron en el año de 1939 en el Texas Highway Institute, para mezclas asfálticas por el método de prueba y error [4]. Más adelante, un equipo similar fue utilizado por el cuerpo de ingenieros GMT en la Segunda Guerra Mundial y en Francia se construyó el compactador giratorio LCPC. El Highway Research Program (SHRP) creado en 1987, inició las investigaciones de compactación giratoria con cementos asfálticos, mas adelante, en la década de los noventa, estudios realizados con mezclas asfálticas condujeron a determinar las especificaciones de la metodología SUPERPAVE, la cuales se presentan en la Tabla 2. Las acciones simultáneas que se aplican al material sometido a un ensayo de compactación giratoria se ilustran en la Figura 3, los valores corresponden a la especificación de la metodología Superpave.

Tabla 2. Especificaciones de la metodología superpave

\begin{tabular}{|c|c|}
\hline Parámetro & Rango o valor especificado \\
\hline Presión vertical & $600 \mathrm{kPa}$ \\
\hline Angulo de giro & $1.25^{\circ}$ \\
\hline Velocidad de giro & $30 \mathrm{rpm}$ \\
\hline
\end{tabular}

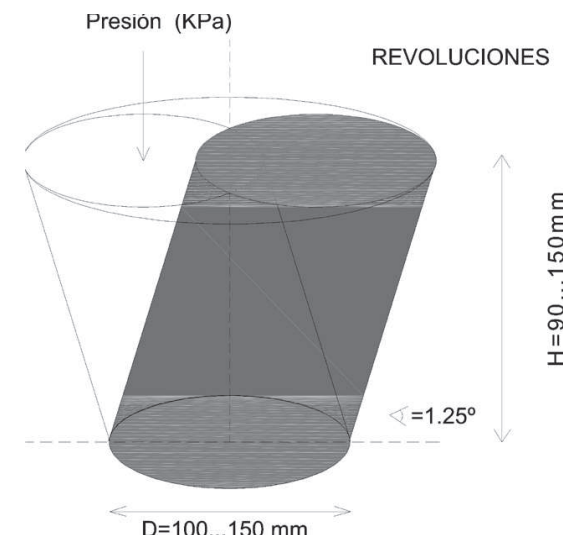

Figura 3. Acciones ejercidas por el equipo de compactación giratoria 
En el año de 1962 los Ingenieros de la Armada de los Estados Unidos estudiaron la compactación giratoria de materiales utilizados en la conformación de subbases y bases granulares, trabajo en el que se obtuvieron las siguientes conclusiones: el método de compactación por impacto es poco recomendado para suelos no cohesivos, el incremento en la presión vertical produce pesos unitarios mayores y el ángulo de giro con el que se deben compactar los materiales deben estar entre $1^{\circ}$ y $2^{\circ}[10]$.

Una de las investigaciones más destacadas, en la que se estudiaron los efectos de la compactación giratoria en un suelo, fue desarrollada por el Departamento de Ingeniería Civil y Ambiental de la Universidad de Florida. En este trabajo se realizaron pruebas de compactación en campo, en tres lugares diferentes, para cada sitio se utilizaron dos niveles de energía de compactación y diferentes contenidos de humedad, el estado de compactación del material se determinó mediante la pruebas con densímetro nuclear. Luego se procedió a la ejecución de los ensayos de compactación vibratoria, compactación por impacto y compactación giratoria en el laboratorio [10]. En este trabajo de investigación se concluyó que:

- El método de compactación por impacto no es el más adecuado para arenas y suelos no cohesivos, debido a que los resultados en campo arrojaron pesos unitarios superiores a los que se obtienen en el laboratorio y las humedades en campo eran menores a las de las pruebas de laboratorio.

- $\quad$ El método de laboratorio que arrojó resultados más cercanos a los obtenidos en campo, fue el método de compactación giratoria.

- Para obtener el peso unitario máximo del suelo estudiado se debe trabajar a una presión vertical de $200 \mathrm{kPa}$, con $1.25^{\circ}$ de inclinación y 90 giros a una velocidad de 20 giros por minuto.

Por otro lado, en el año 2001, el Centro de Desarrollo e Investigación de Ingeniería de la Armada de los Estados Unidos, preparó probetas para ensayos de compresión inconfinada (de $102 \mathrm{~mm}$ de diámetro y $152 \mathrm{~mm}$ de altura), para realizar estudios de compactación giratoria. Los resultados establecieron que para que las probetas obtuvieran la densidad seca máxima, resultado del ensayo de proctor, las muestras debían someterse a un ángulo de giro de $1.25^{\circ}$, una presión de $870 \mathrm{kPa}$ y 90 revoluciones [11].

\section{METODOLOGÍA EXPERIMENTAL}

Previo a la ejecución de los ensayos de compactación giratoria, se realizaron ensayos de caracterización del material de los cuales se obtuvieron los siguientes resultados:

Límites de Atterberg: estos ensayos se realizaron de acuerdo con los procedimientos indicados en las normas INV E - 125 e INV E - 126; se obtuvo un límite líquido igual a 22\%, un límite plástico de $13 \%$ y un índice de plasticidad del $9 \%$.

Granulometría: en la Figura 4 se observa la distribución granulométrica del material utilizado en esta investigación; el $90.20 \%$ de las partículas pasan el tamiz No. 4 y el $38.20 \%$ 
tienen un diámetro inferior a $0.074 \mathrm{~mm}$. De acuerdo con estos valores y la clasificación unificada de suelos (USC), se determinó que el material clasifica dentro del grupo de las arenas limosas. Este ensayo se realizó según lo indicado en la norma INV E - 123.

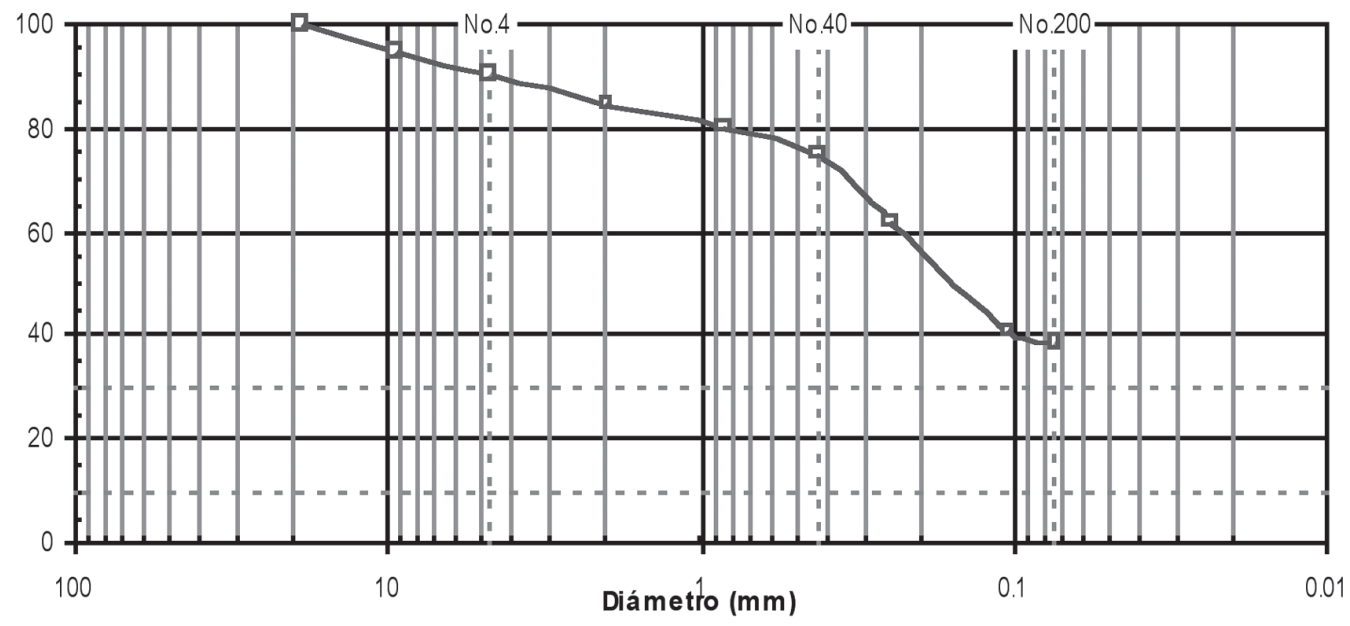

Figura 4. Granulometría del material de granular

Gravedad específica: se realizó la gravedad específica del material de acuerdo con las normas INV E - 128, INV E - 222 e INV E - 223, el valor de gravedad específica de los sólidos del material es 2.65 , valor requerido para obtener la curva teórica de cero vacíos del material.

Proctor modificado y Proctor estándar: estos ensayos se ejecutaron como lo indican las normas INV E - 141 y INV E - 142, resultados de los que se obtuvieron las curvas peso unitario seco vs. Contenido de humedad que se ilustran en la Figura 5.

Una vez realizada la caracterización del material se realizaron las pruebas de compactación en el equipo de compactación giratorio Rainhart's SHRP de la Universidad Militar Nueva Granada, trabajo en el que se establecieron los siguientes parámetros.

- Números de giros: todos los ensayos se hicieron hasta alcanzar los 300 giros. Como el equipo tiene un sensor de desplazamiento vertical, es posible conocer la altura de la muestra en cada giro.

- Ángulo de giro: se trabajó con un ángulo constante de $1.25^{\circ}$.

- Velocidad de rotación: este parámetro depende de las características propias del equipo, para este caso el equipo utilizado trabaja con $30 \mathrm{rpm}$.

- Presión vertical: se trabajó con presiones de 100 kPa, 150 kPa, 200 kPa, 300 kPa, 350 kPa y $400 \mathrm{kPa}$.

- Contenido de humedad: para cada presión de compactación se prepararon muestras con cinco contenidos diferentes de humedad, para un total de 30 muestras. 


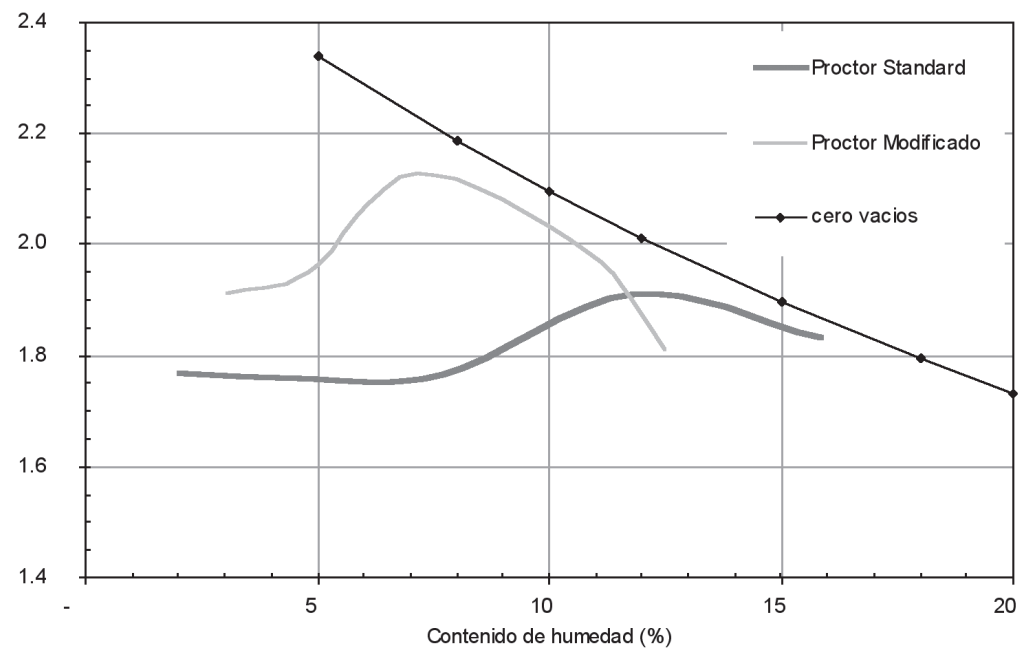

Figura 5. Curvas de Compactación - Ensayos de Proctor Estándar y Modificado.

Según los parámetros definidos anteriormente fue necesario realizar un total de 30 pruebas, luego de la ejecución de cada prueba se obtuvieron probetas como la ilustrada en la Figura 6.

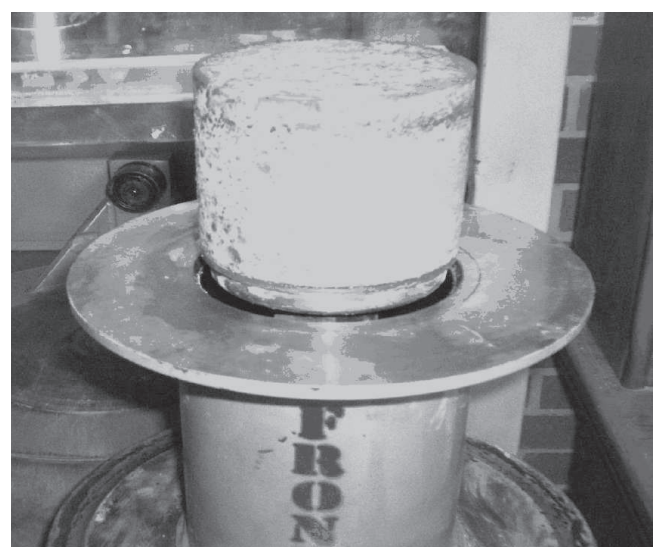

Figura 6. Probeta obtenida luego de finalizada la prueba

Durante la ejecución de los ensayos el programa traza una gráfica en que se representa la altura de la muestra en función del número de giros. Una vez éste finaliza, se obtiene un archivo de datos con la siguiente información: número de giro, altura de la muestra, ángulo de giro y presión vertical. Luego se procede a determinar la humedad final de la muestra y como el diámetro es constante y la altura de la muestra la registra el programa, es posible calcular el peso unitario seco para cada uno de los puntos. Los resultados que se presentan a continuación se dan en función del peso unitario seco y el contenido de humedad. 


\section{ANÁLISIS DE RESULTADOS}

Todos los ensayos realizados en el equipo de compactación giratoria registran en un archivo de texto para cada número de giro la altura de la muestra, la presión vertical y el ángulo de giro. La altura de la muestra permite determinar el volumen de ésta y con el peso y el contenido de humedad, que se toman al finalizar el ensayo, es posible llegar a conocer el peso unitario seco en función del número de giros (ver Figura 7).

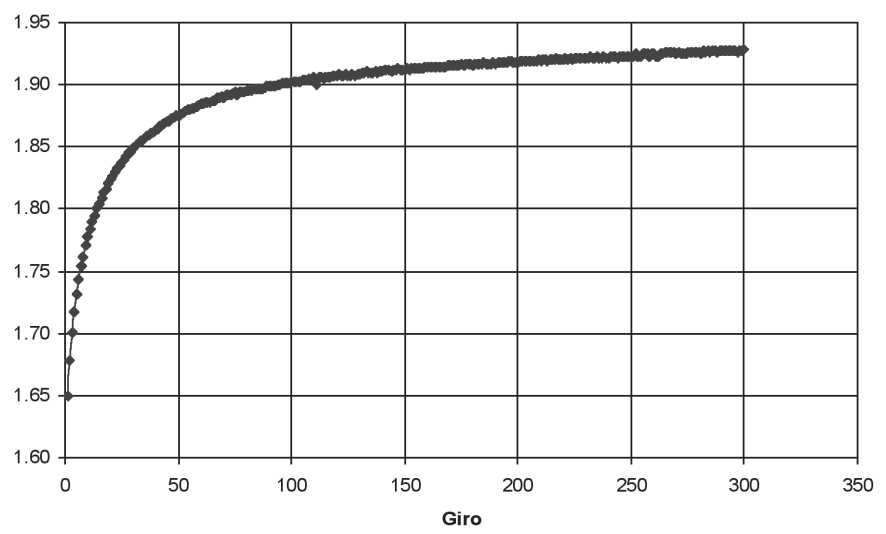

Figura 7. Gráfico del Peso Unitario Seco en función del número de giros para un ensayo de Presión de $100 \mathrm{kPa}$ y humedad de $2.5 \%$

Lo anterior conduce a determinar las variaciones del peso unitario seco en función de los diferentes contenidos de humedad, ver figuras 8, 9, 10, 11 y 12, para cada una de los ensayos realizados a presiones diferentes. En los siguientes gráficos es posible comparar como cambia el peso unitario seco de acuerdo con el número de giros y además se comparan las curvas producto de estos ensayos con las curvas obtenidas en los ensayos de proctor estándar y proctor modificado.

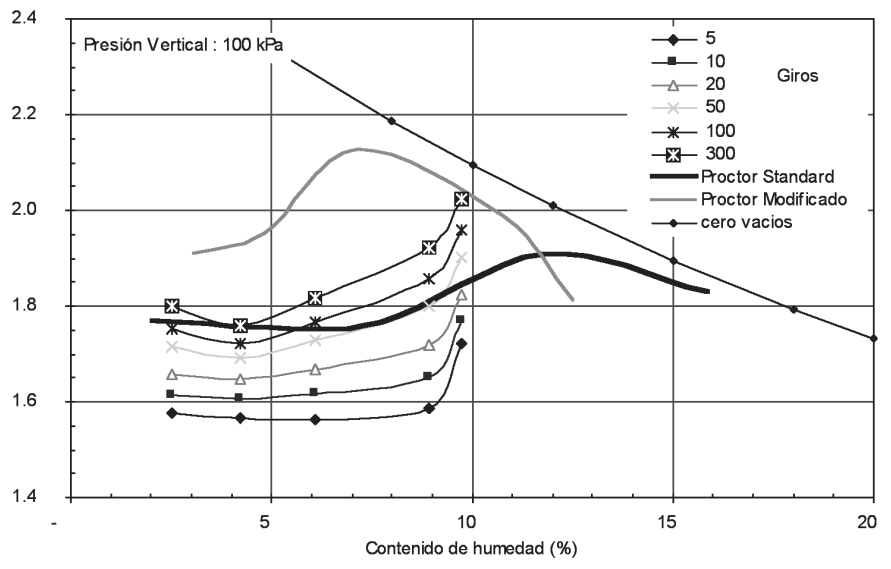

Figura 8. Presión vertical de $100 \mathrm{kPa}$ 


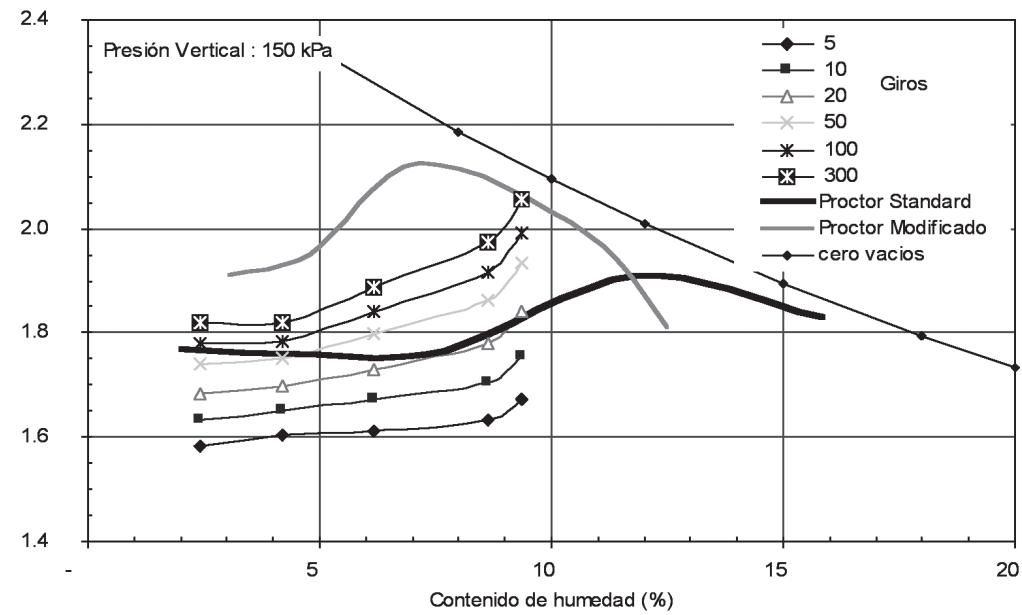

Figura 9. Presión de vertical de $150 \mathrm{kPa}$

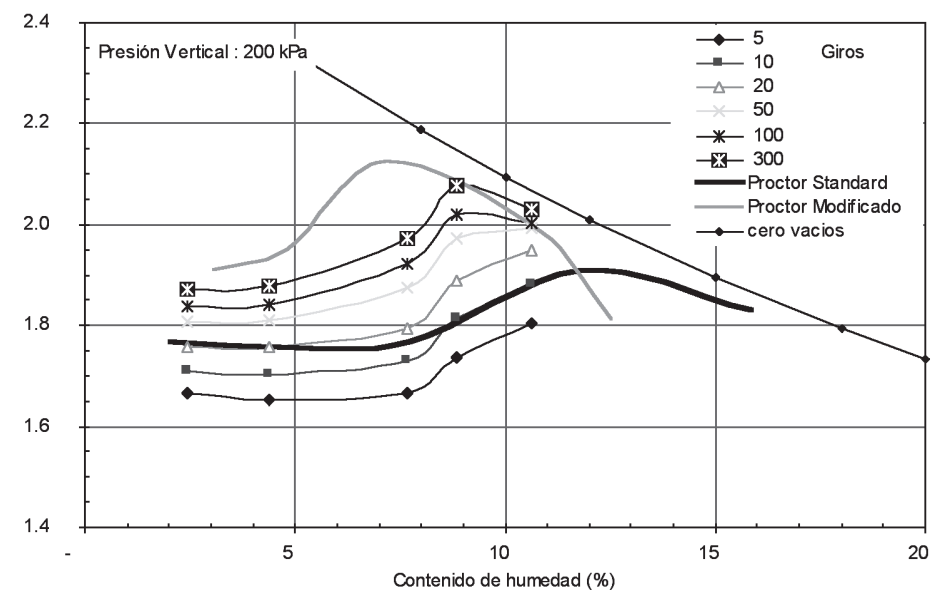

Figura 10. Presión de vertical de $200 \mathrm{kPa}$

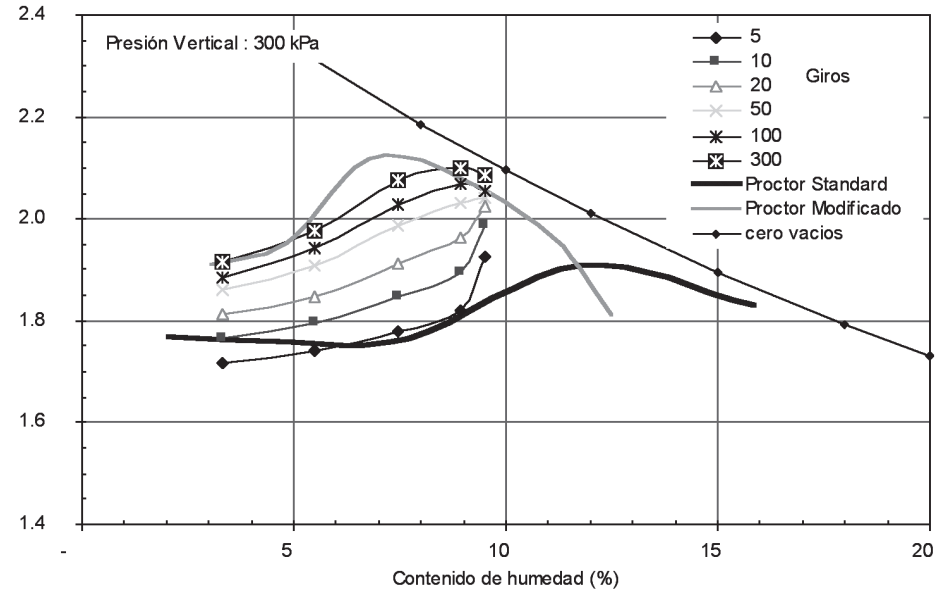

Figura 11. Presión de vertical de $300 \mathrm{kPa}$ 


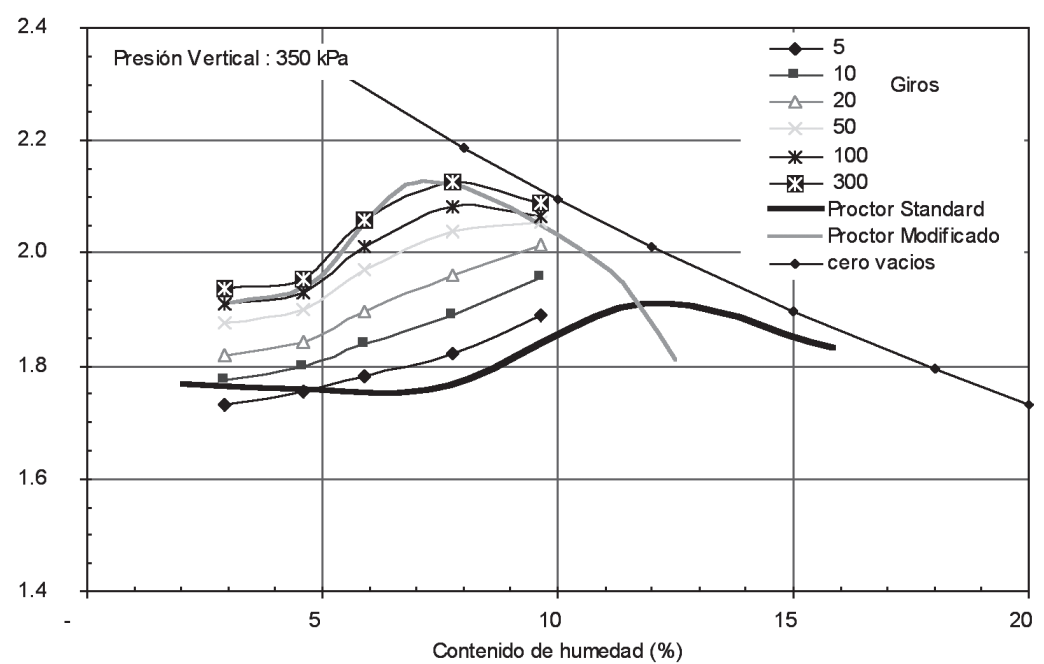

Figura 12. Presión de vertical de $350 \mathrm{kPa}$

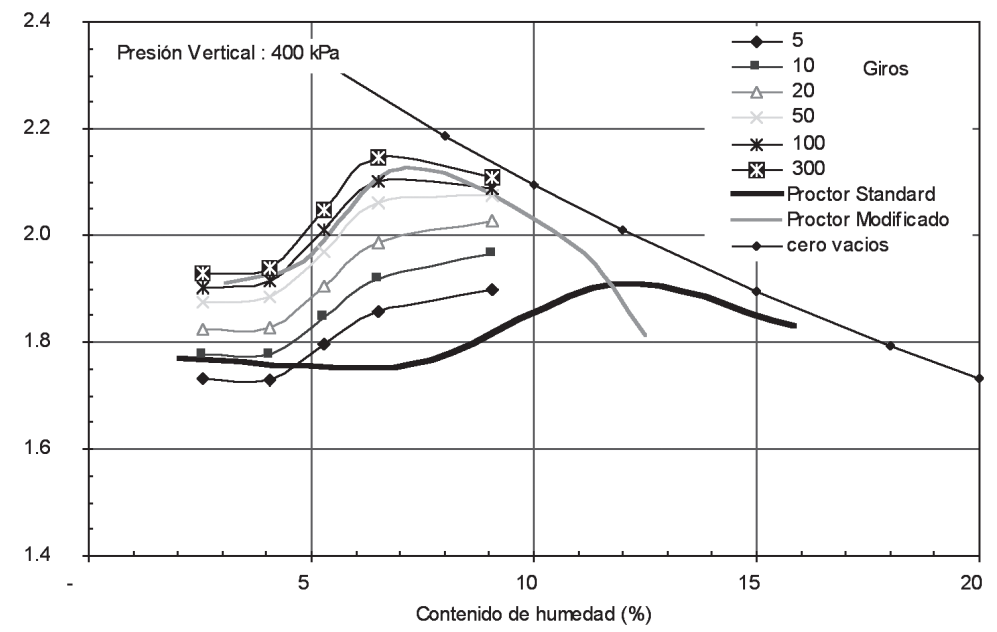

Figura 13. Presión de vertical de $400 \mathrm{kPa}$

Comparando el grupo de ensayos en cada figura, puede observarse que la presión vertical es el principal factor que contribuye a obtener un mayor grado de compactación. Para presiones verticales inferiores a $200 \mathrm{kPa}$, existe una relación creciente entre el contenido de humedad y el peso unitario seco. Solo a partir de $200 \mathrm{kPa}$, se registra un máximo en la curva, es decir se puede identificar un punto óptimo en el contenido de humedad.

Al tratar de utilizar mayores contenidos de humedad, se observó que la presión aplicada junto con el amasado produce drenaje parcial del agua, posiblemente debido a la permeabilidad del material y al ángulo de giro utilizado. El drenaje de las muestras en compactaciones a humedades altas fue un fenómeno que también se presentó en la investigación realizada por el departamento de Ingeniería Civil de la Universidad de Florida [10]. 
De acuerdo con las figuras 12 y 13 se observa que en dos casos es posible alcanzar curvas similares a la obtenida mediante el ensayo de proctor modificado usando el equipo de compactación giratoria: estos son para una presión vertical de $350 \mathrm{kPa}$ y 300 giros, o para una presión vertical de $400 \mathrm{kPa}$ y 100 giros. Esta comparación solo puede considerarse válida para el tipo de material analizado. Una relación general solo puede obtenerse bajo un estudio más amplio en el que se experimente con otros tipos de gradación y plasticidad de los finos.

Para obtener curvas similares a la curva de compactación normal, es necesario utilizar un ángulo de giro menor al utilizado en este estudio.

\section{CONCLUSIONES}

Se propuso un método alternativo en laboratorio para estudiar las relaciones peso unitario - contenido de humedad, utilizando el compactador giratorio. Para demostrar su potencial utilización se realizaron ensayos de compactación proctor y giratoria sobre un material granular.

Además de simular mejor el proceso de compactación en campo, el método de compactación giratoria, es mas versátil, permite un mayor control de las variables utilizadas y es más rápido que el método convencional.

Durante el transcurso de cada ensayo se observó que las mayores variaciones de altura y de peso unitario seco se presentan en los primeros 100 giros. Este comportamiento se evidencia en la Figura 7, donde para los últimos 100 giros las variaciones en altura no fueron significativas y la tendencia de la curva es lineal.

El proceso de compactación giratoria no presenta buenos resultados cuando se utiliza baja presión vertical y el material tiene un contenido de humedad superior al óptimo, puesto que parte del agua sale de la muestra. Este comportamiento deberá ser estudiado en diferentes tipos de suelo.

Se obtuvieron curvas semejantes a las del proctor modificado cuando se utilizó una presión vertical de $350 \mathrm{kPa}, 300$ giros y un ángulo de $1.25^{\circ}$ y para una presión vertical de $400 \mathrm{kPa}$, 100 giros y un ángulo de $1.25^{\circ}$. Esta aproximación se considera válida para el tipo de material utilizado, siendo necesario extender el estudio a otro tipo de materiales.

Es necesario desarrollar un programa de ensayos más extenso para incluir el efecto de otras variables como el ángulo de giro o el número de capas, utilizando diferentes tipos de material para poder formular un procedimiento general que regule el ensayo.

\section{AGRADECIMIENTOS}

El presente trabajo se realizó dentro del proyecto de investigación denominado "Optimización del uso de Geo-Stab y metodología para su evaluación” desarrollado por el Grupo 
de Investigación en Geotecnia de la Universidad Militar Nueva Granada y financiado por Colciencias, y Química Cros S.A. (Contrato 484-2005).

\section{REFERENCIAS}

[1] BRAJA M.D., (1999), Principios de la Ingeniería de Cimentaciones, México, Thomson Editores, 764-777.

[2] BERRY P. y REID D., (2002), Mecánica de Suelos, Bogotá, Mc Graw Hill, 378-383.

[3] CAMACHO J. (2007), Proyecto 1123-08-17492. Informe técnico de avance. Bogotá. $27 \mathrm{p}$.

[4] GARNICA P., GÓMEZ J. y ALAMILLA H., (2003), Algunos aspectos de la densificación de mezclas asfálticas con el compactador giratorio, en: Secretaría de Comunicaciones y Transportes, Instituto mexicano del transporte, Vol. 228, 69 p.

[5] GARNICA P., ALAMILLA H., LÓPEZ J., (2004), Análisis de la influencia de método de compactación en el comportamiento mecánico de mezclas asfálticas, en: Secretaría de Comunicaciones y Transportes, Instituto mexicano del transporte, Vol. 255, 46 p.

[6] GONZÁlEZ L., FERRE M., ORTUÑO L. y OTEO C., (2004), Ingeniería Geológica, Madrid (España), Pearson Prentice Hall, 591 - 592.

[7] HOLTZ R., KOVACS W., (1981), An Introduction to Geotechnical Engineering, United States of America, Prentice Hall Inc, 109 -153 p.

[8] INSTITUTO NACIONAL DE VIAS (1998), Normas de ensayos de materiales para carreteras.

[9] JUÁREZ E. y RICO A., (2000), Mecánica de Suelos Tomo I Fundamentos de la mecánica de suelos, Limusa Noriega Editores, México, 575-612.

[10] RODRÍGUEZ A., DEL CASTILLO H., (1992), Consideraciones sobre compactación de suelos en obras de infraestructura de transporte, en: Secretaria de comunicaciones y transportes instituto mexicano del transporte, Vol. 7, $137 \mathrm{p.}$

[11] SANTONI R., TINGLE J. y WEBSTER S., (2001), Nontraditional Stabilization of Silty - Sand, En: http://www.midwest-equestrian.com/productmaterials/SSEngBen.pdf

[12] PING W.V., XING G., LEONARD. M y YANG Z., (2003), Evaluation of Laboratory Compaction Techniques for Simulating Field Soil Compaction, Department of Civil \& Environmental Engineering Florida A\&M University - Florida State University, 203 p. 
